\title{
Power Hamza Distribution with Application to Lifetime Data
}

\author{
Samuel U. Enogwe ${ }^{1^{*}}{ }^{(0)}$, Chike H. Nwankwo ${ }^{2}$ (), Eric U. Oti ${ }^{3}$ 다 \\ ${ }^{1}$ Department of Statistics, Michael Okpara University of Agriculture, Umudike, Nigeria \\ ${ }^{2}$ Department of Statistics, Nnamdi Azikiwe University, Awka, Nigeria \\ ${ }^{3}$ Department of Statistics, Federal Polytechnic, Ekowe, Nigeria \\ Email: *senogwe@yahoo.com, *enogwe.samuel@mouau.edu.ng
}

How to cite this paper: Enogwe, S.U., Nwankwo, C.H. and Oti, E.U. (2022) Power Hamza Distribution with Application to Lifetime Data. Journal of Applied Mathematics and Physics, 10, 31-48.

https://doi.org/10.4236/jamp.2022.101004

Received: October 7, 2021

Accepted: January 8, 2022

Published: January 11, 2022

Copyright $\odot 2022$ by author(s) and Scientific Research Publishing Inc. This work is licensed under the Creative Commons Attribution International License (CC BY 4.0).

http://creativecommons.org/licenses/by/4.0/

\begin{abstract}
In this paper, a three-parameter lifetime distribution named power Hamza distribution $(\mathrm{PH})$ is proposed. The $\mathrm{PH}$ distribution is a useful generalization of the Hamza distribution which accommodates heavy-tailed, upside-down bathtub and J-shaped hazard rates making it more flexible than the Hamza distribution for modelling various kinds of lifetime data. A comprehensive account of the properties of this distribution is presented. The maximum likelihood estimators of the unknown model parameters are discussed. Finally, a real-life data is analyzed for illustrative purpose proving that the $\mathrm{PH}$ outperforms the Hamza distribution and several other lifetime distributions.
\end{abstract}

\section{Keywords}

Hamza Distribution, Hazard Rate Function, Conditional Moments, Stochastic Ordering, Maximum Likelihood Method

\section{Introduction}

The Hamza distribution was introduced by [1] with the cumulative distribution function (cdf) and the corresponding probability density function (pdf) respectively given by

$$
F_{H}(y)=1-\left[1+\frac{\theta x\left(\theta^{5} y^{5}+6 \theta^{4} y^{4}+30 \theta^{3} y^{3}+120 \theta^{2} y^{2}+360 \theta y+720\right)}{\left(\alpha \theta^{5}+120\right)}\right] \mathrm{e}^{-\theta y}
$$

and

$$
f_{H}(x)=\frac{\theta^{6}}{\alpha \theta^{5}+120}\left(\alpha+\frac{\theta}{6} y^{6}\right) \mathrm{e}^{-\theta y}
$$


for $y>0, \theta>0$ and $\alpha>0$.

It may be observed that the Hamza distribution was obtained by compounding the exponential distribution having scale parameter $\theta$ and the gamma distribution having shape parameter 7 and scale parameter $\theta$, with mixing proportions $p=\alpha \theta^{5} /\left(\alpha \theta^{5}+120\right)$ and $q=\alpha \theta^{5} /\left(\alpha \theta^{5}+120\right)$ such that $p+q=1$.

[1] studied the properties and applications of this distribution in the context of lifetime analysis, showing that the distribution is superior to Lindley distribution due to [2], Ishita distribution by [3] and Pranav distribution by [4], respectively.

The aim of this paper is to introduce a new distribution, called the power Hamza distribution, which is a direct generalization of the Hamza distribution. Some of the distributions proposed using the power transformation include the power Lindley due to [5], power Akash and Shanker proposed by [6] [7], power Ishita and power Aradhana due to [8] [9], power Rama and power Garima due to Abebe et al. [10] [11], power Pranav due to [12], power Sujatha by [13], power Prakaamy by [14]. From the literature reviewed in this paper, all the power transformed distributions were shown to be more flexible than their corresponding baseline distributions and more useful for analyzing complex data structures in various fields of life.

The rest of the paper is organized as follows. The pdf, cdf and hazard rate function of the new distribution is given in Section 2. Section 3 provides a comprehensive account of the properties of the distribution including the moment generating function, moments, skewness, kurtosis, mean residual lifetime, mean deviations, Bonferroni and Lorenz curves, stochastic ordering, entropy measure, stress-strength reliability, distributions and moments of order statistics. In Section 4, the maximum likelihood estimates of the parameters of the distribution are given. Also, Section 5 gives the asymptotic confidence intervals. Section 6 illustrates the proposed model in two real-datasets. The paper is concluded in Section 7.

\section{The Power Hamza (PH) Distribution}

The probability density function, cumulative distribution function and hazard function of the power Hamza distribution having parameters $\theta, \alpha$ and $\beta$ are provided in as Propositions 1, 2 and 3.

Proposition 1. A random variable $X$ is said to have a $P H$ distribution if its $p d f$ is of the form

$$
f_{P H}(x)=\frac{\beta \theta^{6}}{\alpha \theta^{5}+120}\left(\alpha+\frac{\theta}{6} x^{6 \beta}\right) x^{\beta-1} \mathrm{e}^{-\theta x^{\beta}} ; x>0, \theta>0, \alpha>0, \beta>0
$$

Proof. Given the distribution of the Hamza random variable $Y$ defined in (2). Assume that another random variable $X$ is related to $Y$ by the power function $X=g(Y)=Y^{1 / \beta}$. Then the distribution of $X$ is the power Hamza distribution. To derive the distribution of $X$, we notice that $X$ is a one-to-one function of $Y$ and so, $x=0$ when $y=0$ and $x=\infty$ when $y=\infty$, which implies that the support of the distribution of $X$ is $(0, \infty)$. 
Letting $x=g(y)=y^{1 / \beta}$, and $y=g^{-1}(x)=x^{\beta}$ in (2), gives

$$
f_{H}\left(g^{-1}(x)\right)=\frac{\theta^{6}}{\alpha \theta^{5}+120}\left(\alpha+\frac{\theta}{6} x^{6 \beta}\right) \mathrm{e}^{-\theta x^{\beta}}, x>0
$$

According to [15], the probability density function of a continuous random variable $X=g(Y)$ is gotten by

$$
f(x)=f\left(g^{-1}(x)\right)\left|\frac{\mathrm{d} y}{\mathrm{~d} x}\right|
$$

Substituting (4) and $\mathrm{d} y / \mathrm{d} x=\beta x^{\beta-1}$ into (5), one obtains

$$
f_{P H}(x)=\frac{\theta^{6}}{\alpha \theta^{5}+120}\left(\alpha+\frac{\theta}{6} x^{6 \beta}\right) \mathrm{e}^{-\theta x^{\beta}} \times\left|\beta x^{\beta-1}\right| ; x>0
$$

Further simplification of (6) yields the pdf of the power Hamza random variable $X$ defined in (3), and the proof of Proposition 1 is complete.

Corollary 1 . Let $X$ be a power Hamza random variable, then the function defined by Equation (3) is a pdf.

Proof. We show that $f_{P H}(x) \geq 0 \forall x$ and $\int_{0}^{\infty} f_{P H}(x) \mathrm{d} x=1$ are satisfied.

1) $f_{P H}(x) \geq 0 \forall x$

2)

$$
\int_{0}^{\infty} f_{P H}(x) \mathrm{d} x=\int_{0}^{\infty} \frac{\beta \theta^{6}}{\alpha \theta^{5}+120}\left(\alpha+\frac{\theta}{6} x^{6 \beta}\right) x^{\beta-1} \mathrm{e}^{-\theta x^{\beta}} \mathrm{d} x
$$

$$
=\frac{\beta \theta^{6}}{\alpha \theta^{5}+120}\left[\alpha \int_{0}^{\infty} x^{\beta-1} \mathrm{e}^{-\theta x^{\beta}} \mathrm{d} x+\frac{\theta}{6} \int_{0}^{\infty} x^{7 \beta-1} \mathrm{e}^{-\theta x^{\beta}} \mathrm{d} x\right]
$$

By setting $x=y^{1 / \beta}$ in the above integral and noting that

$$
\begin{aligned}
& \int_{0}^{\infty} y^{\alpha-1} \mathrm{e}^{-\theta y} \mathrm{~d} y=\Gamma(\alpha) / \theta^{\alpha} \text { one obtains } \\
& \int_{0}^{\infty} f_{P H}(x) \mathrm{d} x=\frac{\beta \theta^{6}}{\alpha \theta^{5}+120}\left[\frac{\alpha}{\beta} \int_{0}^{\infty} \mathrm{e}^{-\theta y} \mathrm{~d} y+\frac{\theta}{6 \beta} \int_{0}^{\infty} y^{6} \mathrm{e}^{-\theta y} \mathrm{~d} y\right] \\
&=\frac{\beta \theta^{6}}{\alpha \theta^{5}+120}\left[\frac{\alpha}{\beta} \frac{\Gamma(1)}{\theta}+\frac{\theta}{6 \beta} \frac{\Gamma(7)}{\theta^{7}}\right] \\
&=\frac{\beta \theta^{6}}{\alpha \theta^{5}+120}\left[\frac{\alpha}{\beta \theta}+\frac{120}{\beta \theta^{6}}\right] \\
&=\frac{\beta \theta^{6}}{\alpha \theta^{5}+120}\left[\frac{\alpha \theta^{5}+120}{\beta \theta^{6}}\right]=1
\end{aligned}
$$

Henceforth, a random variable $X$ that follows the distribution in (3) is symbolized by $X \sim P H(\theta, \alpha, \beta)$. The power Hamza distribution reduces to the Hamza distribution when $\beta=1$.

Proposition 2. For $x>0, \theta>0, \alpha>0$ and $\beta>0$, the cdf of $X \sim \operatorname{PH}(\theta, \alpha, \beta)$ is given by

$$
F_{P H}(x)=1-\left[1+\frac{\theta x^{\beta}\left(\theta^{5} x^{5 \beta}+6 \theta^{4} x^{4 \beta}+30 \theta^{3} x^{3 \beta}+120 \theta^{2} x^{2 \beta}+360 \theta x^{\beta}+720\right)}{\left(\alpha \theta^{5}+120\right)}\right] \mathrm{e}^{-\theta x^{\beta}}
$$


Proof. [15] defines the cdf of a continuous random variable $X$ as

$$
F(x)=P(X \leq x)=\int_{0}^{x} f(u) \mathrm{d} u
$$

Substituting (3) into (8) leads to

$$
F_{P H}(x)=\frac{\beta \theta^{6}}{\alpha \theta^{5}+120} \int_{0}^{x}\left(\alpha+\frac{\theta}{6} u^{6 \beta}\right) u^{\beta-1} \mathrm{e}^{-\theta u^{\beta}} \mathrm{d} u
$$

Letting $x=u^{1 / \beta}, u=x^{\beta}, \mathrm{d} u / \mathrm{d} x=\beta x^{\beta-1}$, the integral (9) becomes

$$
F_{P H}(x)=\frac{\beta \theta^{6}}{\alpha \theta^{5}+120}\left[\frac{\alpha}{\theta} \int_{0}^{\theta x^{\beta}} \mathrm{e}^{-x} \mathrm{~d} x+\frac{1}{6 \theta^{6}} \int_{0}^{\theta x^{\beta}} x^{6} \mathrm{e}^{-x} \mathrm{~d} x\right]
$$

Applying direct integration to the first part of the square bracket in (10) and integration by parts to the second part the square bracket in (10) gives

$$
\int_{0}^{\theta x^{\beta}} \mathrm{e}^{-x} \mathrm{~d} x=1-\mathrm{e}^{-\theta x^{\beta}}
$$

and

$$
\begin{aligned}
\int_{0}^{\theta x^{\beta}} x^{6} \mathrm{e}^{-x} \mathrm{~d} x= & -\left(\theta x^{\beta}\right)^{6} \mathrm{e}^{-\theta x^{\beta}}-6\left(\theta x^{\beta}\right)^{5} \mathrm{e}^{-\theta x^{\beta}}-30\left(\theta x^{\beta}\right)^{4} \mathrm{e}^{-\theta x^{\beta}} \\
& -120\left(\theta x^{\beta}\right)^{3} \mathrm{e}^{-\theta x^{\beta}}-360\left(\theta x^{\beta}\right)^{2} \mathrm{e}^{-\theta x^{\beta}}-720 \theta x^{\beta} \mathrm{e}^{-\theta x^{\beta}}+720
\end{aligned}
$$

respectively. Substituting (11) and (12) into (10), we obtain

$$
\begin{aligned}
F_{P H}(x)= & \frac{\beta \theta^{6}}{\alpha \theta^{5}+120}\left[\frac{\alpha}{\theta}\left(1-\mathrm{e}^{-\theta x^{\beta}}\right)+\frac{1}{6 \theta^{6}}\left(-\left(\theta x^{\beta}\right)^{6} \mathrm{e}^{-\theta x^{\beta}}-6\left(\theta x^{\beta}\right)^{5} \mathrm{e}^{-\theta x^{\beta}}\right.\right. \\
& -30\left(\theta x^{\beta}\right)^{4} \mathrm{e}^{-\theta x^{\beta}}-120\left(\theta x^{\beta}\right)^{3} \mathrm{e}^{-\theta x^{\beta}}-360\left(\theta x^{\beta}\right)^{2} \mathrm{e}^{-\theta x^{\beta}} \\
& \left.\left.-720 \theta x^{\beta} \mathrm{e}^{-\theta x^{\beta}}+720\right)\right]
\end{aligned}
$$

Further simplification of (13) gives (7), which completes the proof of Proposition 2.

Proposition 3. Let $X \sim P H(\theta, \alpha, \beta)$, then the hazard rate function of $X$ is given by

$$
h_{P H}(x)=\frac{\beta \theta^{6}\left(\alpha+\frac{\theta}{6} x^{6 \beta}\right) x^{\beta-1}}{\left[\theta x^{\beta}\left(\theta^{5} x^{5 \beta}+6 \theta^{4} x^{4 \beta}+30 \theta^{3} x^{3 \beta}+120 \theta^{2} x^{2 \beta}+360 \theta x^{\beta}+720\right)+6\left(\alpha \theta^{5}+120\right)\right]}
$$

Proof. The proof of Proposition 3 follows from using (4) and (7) in the relation

$$
h_{P H}(x)=\frac{f_{P H}(x)}{1-F_{P H}(x)}
$$

Figure 1 and Figure 2 demonstrate the graphs of the pdf and hazard function of the PH distribution for different values of $\theta, \alpha$ and $\beta$. 


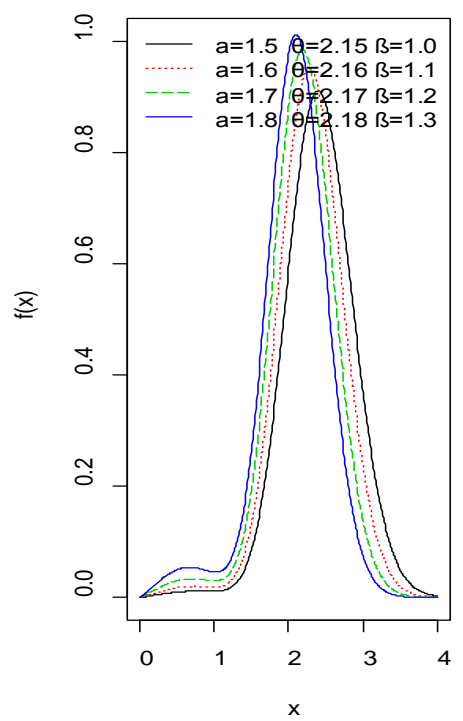

(a)

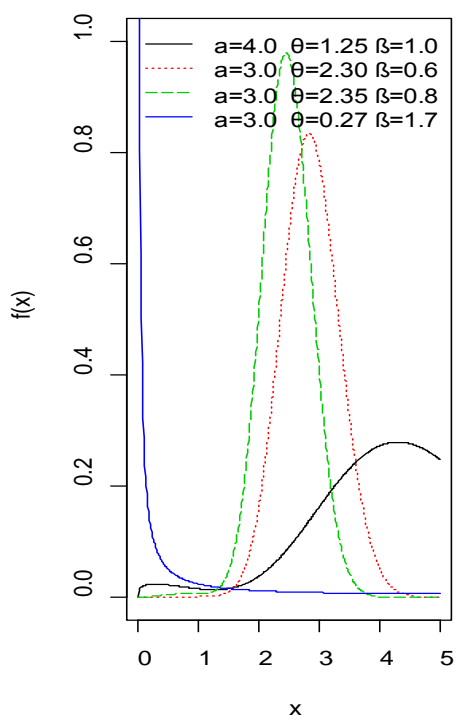

(b)

Figure 1. (a) pdf plot of power hamza distribution; (b) pdf plot of power hamza distribution.

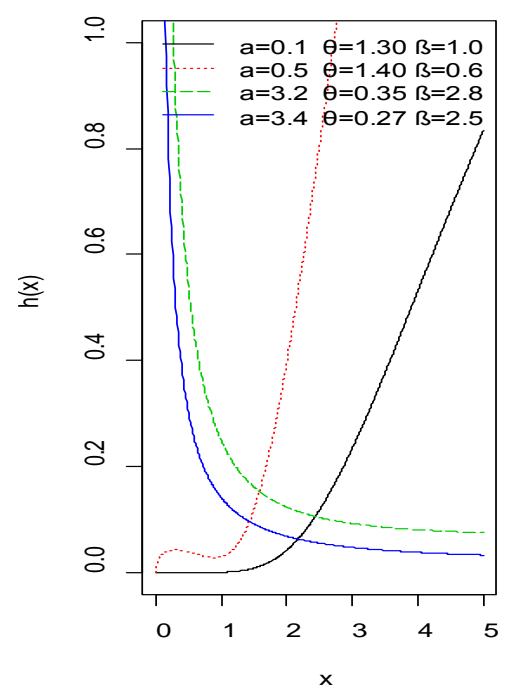

(a)

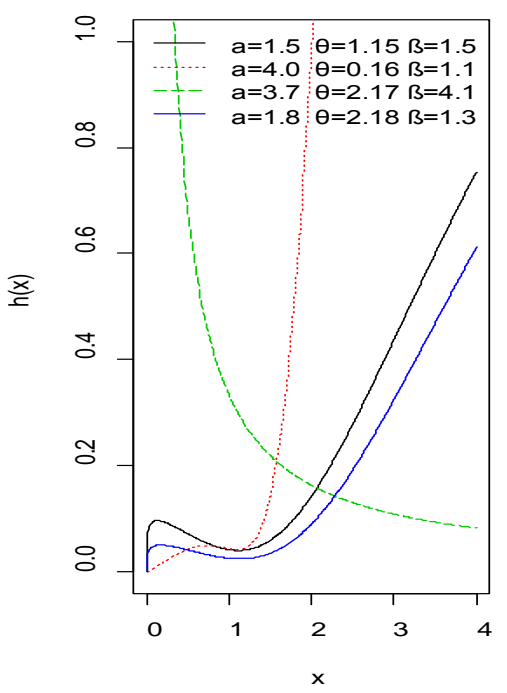

(b)

Figure 2. (a) hazard plot of power hamza distribution; (b) hazard plot of power hamza distribution.

\section{Properties of the Power Hamza (PH) Distribution}

\subsection{Moment Generating Function}

Proposition 4. Let $X \sim P H(\theta, \alpha, \beta)$, then the moment generating function of $X$ is given by

$$
M_{X}(t)=\sum_{r=0}^{\infty} \frac{6 \alpha \theta^{5} \Gamma(r / \beta+1)+\Gamma(r / \beta+7)}{6\left(\alpha \theta^{5}+120\right) r ! \theta^{r / \beta}}\left(\frac{t}{\theta^{1 / \beta}}\right)^{r}
$$

Proof. The moment generating function of $X \sim P H(\theta, \alpha, \beta)$ is obtained as follows 


$$
\begin{aligned}
M_{X}(t) & =E\left(\mathrm{e}^{t X}\right)=\int_{0}^{\infty} \mathrm{e}^{t x} f_{P H}(x) \mathrm{d} x \\
& =\frac{\beta \theta^{6}}{\alpha \theta^{5}+120} \int_{0}^{\infty} \mathrm{e}^{t x}\left(\alpha+\frac{\theta}{6} x^{6 \beta}\right) x^{\beta-1} \mathrm{e}^{-\theta x^{\beta}} \mathrm{d} x \\
& =\frac{\beta \theta^{6}}{\alpha \theta^{5}+120} \sum_{r=0}^{\infty} \frac{t^{r}}{r !}\left[\alpha \int_{0}^{\infty} x^{\beta+r-1} \mathrm{e}^{-\theta x^{\beta}} \mathrm{d} x+\frac{\theta}{6} \int_{0}^{\infty} x^{7 \beta+r-1} \mathrm{e}^{-\theta x^{\beta}} \mathrm{d} x\right]
\end{aligned}
$$

Letting $x=y^{1 / \beta}, y=x^{\beta}$ and $\mathrm{d} x=(1 / \beta) y^{\beta-1} \mathrm{~d} y,(17)$ reduces to

$$
\begin{aligned}
M_{X}(t) & =\frac{\beta \theta^{6}}{\alpha \theta^{5}+120} \sum_{r=0}^{\infty} \frac{t^{r}}{r !}\left[\frac{\alpha}{\beta} \int_{0}^{\infty} y^{r / \beta} \mathrm{e}^{-\theta y} \mathrm{~d} y+\frac{\theta}{6 \beta} \int_{0}^{\infty} x^{r / \beta+6} \mathrm{e}^{-\theta y} \mathrm{~d} y\right] \\
& =\frac{\theta^{6}}{\alpha \theta^{5}+120} \sum_{r=0}^{\infty} \frac{t^{r}}{r !}\left[\frac{\alpha \Gamma(r / \beta+1)}{\theta^{r / \beta+1}}+\frac{\theta \Gamma(r / \beta+7)}{6 \theta^{r / \beta+7}}\right]
\end{aligned}
$$

Further simplification of (18) gives (16), which completes the proof of Proposition 4.

\subsection{Non-Central Moment}

Proposition 5. Let $X \sim P H(\theta, \alpha, \beta)$, then the rth non-moment of $X$ is given by

$$
\mu_{r}^{\prime}=\frac{6 \alpha \theta^{5} \Gamma(r / \beta+1)+\Gamma(r / \beta+7)}{6 \theta^{r / \beta}\left(\alpha \theta^{5}+120\right)} ; r=1,2,3, \cdots
$$

Proof. The $r$ th moment of $X \sim P H(\theta, \alpha, \beta)$ is obtained as follows

$$
\begin{aligned}
\mu_{r}^{\prime} & =E\left(X^{r}\right)=\int_{0}^{\infty} x^{r} f_{P H}(x) \mathrm{d} x=\frac{\beta \theta^{6}}{\alpha \theta^{5}+120} \int_{0}^{\infty} x^{r}\left(\alpha+\frac{\theta}{6} x^{6 \beta}\right) x^{\beta-1} \mathrm{e}^{-\theta x^{\beta}} \mathrm{d} x \\
& =\frac{\beta \theta^{6}}{\alpha \theta^{5}+120}\left[\alpha \int_{0}^{\infty} x^{\beta+r-1} \mathrm{e}^{-\theta x^{\beta}} \mathrm{d} x+\frac{\theta}{6} \int_{0}^{\infty} x^{7 \beta+r-1} \mathrm{e}^{-\theta x^{\beta}} \mathrm{d} x\right]
\end{aligned}
$$

Putting $x=y^{1 / \beta}, \quad y=x^{\beta}$ and $\mathrm{d} x=(1 / \beta) y^{\beta-1} \mathrm{~d} y$ into (20), yields

$$
\begin{aligned}
\mu_{r}^{\prime} & =\frac{\theta^{6}}{\alpha \theta^{5}+120}\left[\alpha \int_{0}^{\infty} y^{\frac{r}{\beta}-1} \mathrm{e}^{-\theta y} \mathrm{~d} y+\frac{\theta_{6}^{\infty}}{6} \int_{0}^{\left(\frac{r}{\beta}+6\right)-1} \mathrm{e}^{-\theta y} \mathrm{~d} y\right] \\
& =\frac{\theta^{6}}{\alpha \theta^{5}+120}\left[\frac{\alpha \Gamma(r / \beta+1)}{\theta^{r / \beta+1}}+\frac{\theta \Gamma(r / \beta+7)}{6 \theta^{r / \beta+7}}\right]
\end{aligned}
$$

Simplifying (21) a little bit, we get (19), hence the proof of Proposition 5.

Corollary 2. The first four non-central moments of $X \sim P H(\theta, \alpha, \beta)$ are

$$
\begin{gathered}
\mu_{1}^{\prime}=\frac{6 \alpha \theta^{5} \Gamma(1 / \beta+1)+\Gamma(1 / \beta+7)}{6 \theta^{1 / \beta}\left(\alpha \theta^{5}+120\right)}=\mu \\
\mu_{2}^{\prime}=\frac{6 \alpha \theta^{5} \Gamma(2 / \beta+1)+\Gamma(2 / \beta+7)}{6 \theta^{2 / \beta}\left(\alpha \theta^{5}+120\right)} \\
\mu_{3}^{\prime}=\frac{6 \alpha \theta^{5} \Gamma(3 / \beta+1)+\Gamma(3 / \beta+7)}{6 \theta^{3 / \beta}\left(\alpha \theta^{5}+120\right)}
\end{gathered}
$$




$$
\mu_{4}^{\prime}=\frac{6 \alpha \theta^{5} \Gamma(4 / \beta+1)+\Gamma(4 / \beta+7)}{6 \theta^{4 / \beta}\left(\alpha \theta^{5}+120\right)}
$$

Proof. The proof of (22)-(25) follows directly from Proposition 5 by substituting $r=1,2,3$ and 4 into (19).

\subsection{Variance}

Apart from the non-central moments, variance of a distribution is always relevant for measuring the spread from the mean. So, we provide the variance of the $\mathrm{PH}$ distribution in Proposition 6.

Proposition 6. Let $X \sim P H(\theta, \alpha, \beta)$, then the variance of $X$ is given by

$$
\sigma^{2}=\frac{6\left(\alpha \theta^{5}+120\right)\left(6 \alpha \theta^{5} \Gamma(2 / \beta+1)+\Gamma(2 / \beta+7)\right)-\left(6 \alpha \theta^{5} \Gamma(1 / \beta+1)+\Gamma(1 / \beta+7)\right)^{2}}{36 \theta^{2 / \beta}\left(\alpha \theta^{5}+120\right)^{2}}
$$

Proof. The variance of a random variable $X$ can be computed using the relation

$$
\sigma^{2}=\mu_{2}^{\prime}-\left(\mu_{1}^{\prime}\right)^{2}
$$

The proof of (26) follows directly from substituting (22) and (23) into (27)

$$
\sigma^{2}=\frac{6 \alpha \theta^{5} \Gamma(2 / \beta+1)+\Gamma(2 / \beta+7)}{6 \theta^{2 / \beta}\left(\alpha \theta^{5}+120\right)}-\left(\frac{6 \alpha \theta^{5} \Gamma(1 / \beta+1)+\Gamma(1 / \beta+7)}{6 \theta^{1 / \beta}\left(\alpha \theta^{5}+120\right)}\right)^{2}
$$

\subsection{Coefficient of Variation and Index of Dispersion}

The coefficient of variation is given by

$$
\begin{gathered}
c v=\frac{\sigma}{\mu}=\frac{\left[\frac{6\left(\alpha \theta^{5}+120\right)\left(6 \alpha \theta^{5} \Gamma(2 / \beta+1)+\Gamma(2 / \beta+7)\right)-\left(6 \alpha \theta^{5} \Gamma(1 / \beta+1)+\Gamma(1 / \beta+7)\right)^{2}}{36 \theta^{2 / \beta}\left(\alpha \theta^{5}+120\right)^{2}}\right]^{1 / 2}}{\frac{6 \alpha \theta^{5} \Gamma(1 / \beta+1)+\Gamma(1 / \beta+7)}{6 \theta^{1 / \beta}\left(\alpha \theta^{5}+120\right)}} \\
=\frac{\left[6\left(\alpha \theta^{5}+120\right)\left(6 \alpha \theta^{5} \Gamma(2 / \beta+1)+\Gamma(2 / \beta+7)\right)-\left(6 \alpha \theta^{5} \Gamma(1 / \beta+1)+\Gamma(1 / \beta+7)\right)^{2}\right]^{1 / 2}}{6 \alpha \theta^{5} \Gamma(1 / \beta+1)+\Gamma(1 / \beta+7)}
\end{gathered}
$$

The index of dispersion is given by

$$
\begin{aligned}
\gamma & =\frac{\sigma^{2}}{\mu}=\frac{\left[\frac{6\left(\alpha \theta^{5}+120\right)\left(6 \alpha \theta^{5} \Gamma(2 / \beta+1)+\Gamma(2 / \beta+7)\right)-\left(6 \alpha \theta^{5} \Gamma(1 / \beta+1)+\Gamma(1 / \beta+7)\right)^{2}}{36 \theta^{2 / \beta}\left(\alpha \theta^{5}+120\right)^{2}}\right]}{\frac{6 \alpha \theta^{5} \Gamma(1 / \beta+1)+\Gamma(1 / \beta+7)}{6 \theta^{1 / \beta}\left(\alpha \theta^{5}+120\right)}} \\
& =\frac{6\left(\alpha \theta^{5}+120\right)\left(6 \alpha \theta^{5} \Gamma(2 / \beta+1)+\Gamma(2 / \beta+7)\right)-\left(6 \alpha \theta^{5} \Gamma(1 / \beta+1)+\Gamma(1 / \beta+7)\right)^{2}}{6 \theta^{1 / \beta}\left(\alpha \theta^{5}+120\right)\left(6 \alpha \theta^{5} \Gamma(1 / \beta+1)+\Gamma(1 / \beta+7)\right)}
\end{aligned}
$$




\subsection{Central Moment}

Proposition 7. Let $X \sim P H(\theta, \alpha, \beta)$, then the central moment of $X$ is given by

$$
\mu_{r}=\sum_{i=0}^{r}\left(\begin{array}{l}
r \\
i
\end{array}\right)(-1)^{r-i}\left(\frac{6 \alpha \theta^{5} \Gamma(1 / \beta+1)+\Gamma(1 / \beta+7)}{6 \theta^{1 / \beta}\left(\alpha \theta^{5}+120\right)}\right)^{r-i} \times\left(\frac{6 \alpha \theta^{5} \Gamma(i / \beta+1)+\Gamma(i / \beta+7)}{6 \theta^{i / \beta}\left(\alpha \theta^{5}+120\right)}\right)
$$

Proof. The $t$ th moment of the $X \sim P H(\theta, \alpha, \beta)$ can be obtained from the relation

$$
\mu_{r}=E(X-\mu)^{r}=\sum_{i=0}^{r}\left(\begin{array}{l}
r \\
i
\end{array}\right)(-\mu)^{r-i} E\left(X^{i}\right)
$$

Using (22) and (19) in (32) gives (31) and the proof of Proposition 7 is complete.

\subsection{Conditional Moment}

A function that is useful in deriving the mean residual life function of a component as well as the mean deviations is the conditional moment. Given that $X$ follows a power Hamza distribution with parameters $\theta, \alpha$ and $\beta$, then

$$
E\left(X^{n} \mid X>x\right)=\frac{1}{(1-F(x))} J_{n}(x)
$$

where

$$
\begin{aligned}
J_{n}(x) & =\int_{x}^{\infty} t^{n} f_{\text {BEI }}(t) \mathrm{d} t=\frac{\beta \theta^{6}}{\alpha \theta^{5}+120} \int_{x}^{\infty} t^{n}\left(\alpha+\frac{\theta}{6} t^{6 \beta}\right) t^{\beta-1} \mathrm{e}^{-\theta t^{\beta}} \mathrm{d} t \\
& =\frac{\beta \theta^{6}}{\alpha \theta^{5}+120}\left[\alpha \int_{x}^{\infty} t^{\beta+n-1} \mathrm{e}^{-\theta t^{\beta}} \mathrm{d} t+\frac{\theta}{6} \int_{x}^{\infty} t^{7 \beta+n-1} \mathrm{e}^{-\theta t^{\beta}} \mathrm{d} t\right]
\end{aligned}
$$

Letting $x=\theta t^{\beta}$ in (34) leads to

$$
\begin{gathered}
J_{n}(x)=\frac{\beta \theta^{6}}{\alpha \theta^{5}+120}\left[\frac{\alpha}{\beta \theta^{\beta / n+1}} \int_{\theta x^{\beta}}^{\infty} x^{(\beta / n+1)-1} \mathrm{e}^{-x} \mathrm{~d} x+\frac{\theta}{6 \beta \theta^{\beta / n+7}} \int_{\theta x^{\beta}}^{\infty} x^{(\beta / n+7)-1} \mathrm{e}^{-x} \mathrm{~d} x\right] \\
=\frac{\beta \theta^{6}}{\alpha \theta^{5}+120}\left[\frac{\alpha}{\beta \theta^{\beta / n+1}} \Gamma\left(\frac{\beta}{n}+1, \theta x^{\beta}\right)+\frac{\theta}{6 \beta \theta^{\beta / n+7}} \Gamma\left(\frac{\beta}{n}+7, \theta x^{\beta}\right)\right] \\
J_{n}(x)=\frac{\alpha \theta^{5} \Gamma\left(\frac{\beta}{n}+1, \theta x^{\beta}\right)+\Gamma\left(\frac{\beta}{n}+7, \theta x^{\beta}\right)}{6\left(\alpha \theta^{5}+120\right) \theta^{\beta / n}}
\end{gathered}
$$

where $\Gamma(a, z)=\int_{x}^{\infty} t^{a-1} \mathrm{e}^{-t} \mathrm{~d} t$ is the complementary incomplete gamma function.

Also from (7), we get

$$
(1-F(x))=\left[1+\frac{\theta x^{\beta}\left(\theta^{5} x^{5 \beta}+6 \theta^{4} x^{4 \beta}+30 \theta^{3} x^{3 \beta}+120 \theta^{2} x^{2 \beta}+360 \theta x^{\beta}+720\right)}{\left(\alpha \theta^{5}+120\right)}\right] \mathrm{e}^{-\theta x^{\beta}}
$$


Plugging (35) and (36) into (33), we have the conditional moment as

$$
E\left(X^{n} \mid X>x\right)=\frac{\left[\alpha \theta^{5} \Gamma\left(\frac{\beta}{n}+1, \theta x^{\beta}\right)+\Gamma\left(\frac{\beta}{n}+7, \theta x^{\beta}\right)\right] \mathrm{e}^{\theta x^{\beta}}}{\left[\theta x^{\beta}\left(\theta^{5} x^{5 \beta}+6 \theta^{4} x^{4 \beta}+30 \theta^{3} x^{3 \beta}+120 \theta^{2} x^{2 \beta}+360 \theta x^{\beta}+720\right)+6\left(\alpha \theta^{5}+120\right)\right] \theta^{\beta / n}}
$$

\subsection{Mean Residual Life Function}

In many life testing experiments, it is always of interest to know the additional lifetime given that a component has survived until a certain amount of time. To achieve this purpose, the mean residual life function (MRL), which refers to the expected remaining life, $X-x$, given that the item has survived up to time $x$, is required. It may be observed from (37), that the MRL function is derived from the conditional as follows

$$
m_{X}(x)=E(X-x \mid X>x)-x
$$

Putting $n=1$ in (37) and substituting the result into (38), we obtain MRL function as

$$
m_{X}(x)=\frac{\left[\alpha \theta^{5} \Gamma\left(\beta+1, \theta x^{\beta}\right)+\Gamma\left(\beta+7, \theta x^{\beta}\right)\right] \mathrm{e}^{\theta x^{\beta}}}{\left[\theta x^{\beta}\left(\theta^{5} x^{5 \beta}+6 \theta^{4} x^{4 \beta}+30 \theta^{3} x^{3 \beta}+120 \theta^{2} x^{2 \beta}+360 \theta x^{\beta}+720\right)+6\left(\alpha \theta^{5}+120\right)\right] \theta^{\beta}}-x
$$

\subsection{Mean Deviations}

In statistical modelling, it is often an interest to measure the spread in a population from either the mean or the median. To achieve this, two indices, namely mean deviation about the mean $\left(\mu_{d}\right)$ and mean deviation about the median $\left(M_{d}\right)$ are used. Let $\mu$ denote the mean and $M$, the median of a power Hamza distributed random variable $X$. The values of $\mu_{d}$ and $M_{d}$ can be calculated using the relationships

$$
\mu_{d}=\int_{0}^{\infty}|x-\mu| f_{P H}(x) \mathrm{d} x=2 \mu F(\mu)-2 \mu+2 J_{1}(\mu)
$$

and

$$
M_{d}=\int_{0}^{\infty}|x-M| f_{P H}(x) \mathrm{d} x=2 J_{1}(M)-\mu
$$

respectively. By replacing $x$ with $\mu$ and $M$ in (7) and (35), yields the following

$$
\begin{array}{r}
\mu_{d}=2 \mu\left[1-\left(1+\frac{\theta \mu^{\beta}\left(\theta^{5} \mu^{5 \beta}+6 \theta^{4} \mu^{4 \beta}+30 \theta^{3} \mu^{3 \beta}+120 \theta^{2} \mu^{2 \beta}+360 \theta \mu^{\beta}+720\right)}{\left(\alpha \theta^{5}+120\right)}\right) \mathrm{e}^{-\theta \mu^{\beta}}\right] \\
-\frac{2\left[6 \mu\left(\alpha \theta^{5}+120\right) \theta^{\beta}-\left(\alpha \theta^{5} \Gamma\left(\beta+1, \theta \mu^{\beta}\right)+\Gamma\left(\beta+7, \theta \mu^{\beta}\right)\right)\right]}{6\left(\alpha \theta^{5}+120\right) \theta^{\beta}} \\
M_{d}=\frac{2\left[\alpha \theta^{5} \Gamma\left(\beta+1, \theta M^{\beta}\right)+\Gamma\left(\beta+7, \theta M^{\beta}\right)-3 \mu\left(\alpha \theta^{5}+120\right) \theta^{\beta}\right]}{3\left(\alpha \theta^{5}+120\right) \theta^{\beta}}
\end{array}
$$




\subsection{Bonferroni and Lorenz Curves}

It has been found that the Bonferroni curve proposed by [16] and Lorenz curve proposed by [17] have applications in the fields of economics, reliability, demography, insurance, medicine, among others. So, if $X \sim P H(\theta, \alpha, \beta)$, the Bonferroni and Lorenz curves are respectively given by

$$
B(p)=\frac{1}{p \mu} \int_{0}^{q} x f_{P H}(x) \mathrm{d} x
$$

and

$$
L(p)=\frac{1}{\mu} \int_{0}^{q} x f_{P H}(x) \mathrm{d} x
$$

where $\mu=E(X)$ and $q=F^{-1}(p), 0<p<1$. Hence, for the power Hamza pdf (3), one gets

$$
\begin{aligned}
\int_{0}^{q} x f_{P H}(x) \mathrm{d} x & =\frac{\beta \theta^{6}}{\alpha \theta^{5}+120} \int_{0}^{q} x\left(\alpha+\frac{\theta}{6} x^{6 \beta}\right) x^{\beta-1} \mathrm{e}^{-\theta x^{\beta}} \mathrm{d} x \\
& =\frac{\beta \theta^{6}}{\alpha \theta^{5}+120}\left[\alpha \int_{0}^{q} x^{\beta} \mathrm{e}^{-\theta x^{\beta}} \mathrm{d} x+\frac{\theta}{6} \int_{0}^{q} x^{7 \beta} \mathrm{e}^{-\theta x^{\beta}} \mathrm{d} x\right]
\end{aligned}
$$

Letting $w=\theta x^{\beta}, \quad x=(w / \theta)^{1 / \beta}, \mathrm{d} x=(1 / \beta)(1 / \theta)^{1 / \beta} w^{1 / \beta-1} \mathrm{~d} w$ and noting that $x \in(0, q)$ implies $w \in\left(0, \theta q^{\beta}\right),(1)$ becomes

$$
\begin{aligned}
& \int_{0}^{q} x f_{P H}(x) \mathrm{d} x \\
& =\frac{\theta^{6}}{\alpha \theta^{5}+120}\left[\alpha\left(\frac{1}{\theta}\right)^{\left(\beta+\frac{1}{\beta}\right)} \int_{0}^{\theta q^{\beta}} w^{\left(\beta+\frac{1}{\beta}\right)-1} \mathrm{e}^{-w} \mathrm{~d} w+\frac{\theta}{6}\left(\frac{1}{\theta}\right)^{\left(7 \beta+\frac{1}{\beta}\right)} \int_{0}^{\theta q^{\beta}} w^{\left(7 \beta+\frac{1}{\beta}\right)-1} \mathrm{e}^{-w} \mathrm{~d} w\right] \\
& =\frac{\theta^{6}}{\alpha \theta^{5}+120}\left[\alpha\left(\frac{1}{\theta}\right)^{\left(\beta+\frac{1}{\beta}\right)} \Gamma\left(\beta+\frac{1}{\beta}, \theta q^{\beta}\right)+\frac{\theta}{6}\left(\frac{1}{\theta}\right)^{\left(7 \beta+\frac{1}{\beta}\right)} \Gamma\left(7 \beta+\frac{1}{\beta}, \theta q^{\beta}\right)\right] \\
& =\frac{\theta^{6}\left(\frac{1}{\theta}\right)^{\frac{\beta^{2}+1}{\beta}}\left[6 \alpha \theta^{6 \beta} \Gamma\left(\frac{\beta^{2}+1}{\beta}, \theta q^{\beta}\right)+\theta \Gamma\left(\frac{7 \beta^{2}+1}{\beta}, \theta q^{\beta}\right)\right]}{6 \theta^{6 \beta}\left(\alpha \theta^{5}+120\right)}
\end{aligned}
$$

Substituting (46) and (47) into (44) and (45), we obtain the Bonferroni and Lorenz curves, respectively, for the power Hamza distribution as

$$
\begin{aligned}
& B(p)=\frac{\theta^{6}\left(\frac{1}{\theta}\right)^{\frac{\beta^{2}+1}{\beta}}\left[6 \alpha \theta^{6 \beta} \Gamma\left(\frac{\beta^{2}+1}{\beta}, \theta q^{\beta}\right)+\theta \Gamma\left(\frac{7 \beta^{2}+1}{\beta}, \theta q^{\beta}\right)\right]}{6 p \theta^{6 \beta} \mu\left(\alpha \theta^{5}+120\right)} \\
& L(p)=\frac{\theta^{6}\left(\frac{1}{\theta}\right)^{\frac{\beta^{2}+1}{\beta}}\left[6 \alpha \theta^{6 \beta} \Gamma\left(\frac{\beta^{2}+1}{\beta}, \theta q^{\beta}\right)+\theta \Gamma\left(\frac{7 \beta^{2}+1}{\beta}, \theta q^{\beta}\right)\right]}{6 \theta^{6 \beta} M\left(\alpha \theta^{5}+120\right)}
\end{aligned}
$$




\subsection{Stochastic Ordering}

In this section, we discuss the comparative behaviour of the power Hamza random variable using the stochastic ordering. In line with [18], a power Hamza random variable $X$ is said to be smaller than another random variable $Y$ in the 1) stochastic order $\left(X \leq_{s t} Y\right)$ if $\left.F_{X}(x) \geq F_{Y}(x) \forall x, 2\right)$ hazard rate order $\left(X \leq{ }_{h r} Y\right)$ if $\left.h_{X}(x) \geq h_{Y}(x) \forall x, 3\right)$ mean residual life order $\left(X \leq_{m r l} Y\right)$ if $m_{X}(x) \geq m_{Y}(x) \forall x$ and 4) likelihood ratio order $\left(X \leq{ }_{l r} Y\right)$ if $f_{X}(x) / f_{Y}(x)$ a decreasing function of $x$. To show the flexibility of the power Hamza distribution, we present the following Proposition.

Proposition 8. Let $X \sim\left(\theta_{1}, \alpha_{1}, \beta_{1}\right)$ and $Y \sim\left(\theta_{2}, \alpha_{2}, \beta_{2}\right)$ be two independent random variables. If 1) $\theta_{1}>\theta_{2}, \alpha_{1}=\alpha_{2}$ and $\beta_{1}=\beta_{2}$; 2) $\alpha_{1}>\alpha_{2}$, $\theta_{1}=\theta_{2}, \quad \beta_{1}=\beta_{2}$; 3) $\beta_{1}<\beta_{2}, \theta_{1}=\theta_{2}, \alpha_{1}=\alpha_{2}$ and 4) $\theta_{1}>\theta_{2}, \alpha_{2}<\alpha_{1}$, $\beta_{1}<\beta_{2}$, then $X \leq_{l r} Y, X \leq_{h r} Y, X \leq_{m l r} Y$ and $X \leq_{s t} Y$.

Proof. The likelihood ratio is

$$
\left.\frac{f_{X}\left(x ; \theta_{1}, \alpha_{1}, \beta_{1}\right)}{f_{Y}\left(x ; \theta_{2}, \alpha_{2}, \beta_{2}\right)}=\frac{\beta_{1} \theta_{1}^{6}\left(\alpha_{2} \theta_{2}^{5}+120\right)}{\beta_{2} \theta_{2}^{6}\left(\alpha_{1} \theta_{1}^{5}+120\right)} x^{\beta_{1}-\beta_{2}}\left(\frac{6 \alpha_{1}+\theta_{1} x^{6 \beta_{1}}}{6 \alpha_{2}+\theta_{2} x^{6 \beta_{2}}}\right) \mathrm{e}^{-\left(\theta_{1} x^{\beta_{1}}-\theta_{2} x^{\beta_{2}}\right.}\right)
$$

The log-likelihood ratio is

$$
\begin{aligned}
\ln \left\{\frac{f_{X}\left(x ; \theta_{1}, \alpha_{1}, \beta_{1}\right)}{f_{Y}\left(x ; \theta_{2}, \alpha_{2}, \beta_{2}\right)}\right\}= & \ln \left[\frac{\beta_{1} \theta_{1}^{6}\left(\alpha_{2} \theta_{2}^{5}+120\right)}{\beta_{2} \theta_{2}^{6}\left(\alpha_{1} \theta_{1}^{5}+120\right)}\right]+\left(\beta_{1}-\beta_{2}\right) \ln x \\
& +\ln \left(\frac{6 \alpha_{1}+\theta_{1} x^{6 \beta_{1}}}{6 \alpha_{2}+\theta_{2} x^{6 \beta_{2}}}\right)-\left(\theta_{1} x^{\beta_{1}}-\theta_{2} x^{\beta_{2}}\right)
\end{aligned}
$$

Differentiating the log-likelihood with respect to $x$, we get

$$
\begin{aligned}
& \frac{\mathrm{d}}{\mathrm{d} x} \ln \left\{\frac{f_{X}\left(x ; \theta_{1}, \alpha_{1}, \beta_{1}\right)}{f_{Y}\left(x ; \theta_{2}, \alpha_{2}, \beta_{2}\right)}\right\} \\
& =\frac{36\left(6 \theta_{1} \alpha_{2} \beta_{1} x^{6 \beta_{1}-1}+\theta_{2} \alpha_{1} \beta_{2} x^{6 \beta_{2}-1}\right)+6 \theta_{1} \theta_{2}\left(\beta_{1}-\beta_{2}\right) x^{6\left(\beta_{1}-\beta_{2}\right)-1}}{\left(6 \alpha_{1}+\theta_{1} x^{6 \beta_{1}}\right)\left(6 \alpha_{2}+\theta_{2} x^{6 \beta_{2}}\right)} \\
& +\frac{\left(\beta_{1}-\beta_{2}\right)}{x}-\left(\theta_{1} \beta_{1} x^{\beta_{1}-1}-\theta_{2} \beta_{2} x^{\beta_{2}-1}\right)
\end{aligned}
$$

Since $\frac{\mathrm{d}}{\mathrm{d} x} \ln \left\{\frac{f_{X}\left(x ; \theta_{1}, \alpha_{1}, \beta_{1}\right)}{f_{X}\left(x ; \theta_{1}, \alpha_{1}, \beta_{1}\right)}\right\}<0$ for conditions 1), 2), 3) and 4), then $X \leq_{l r} Y$ and hence, $X \leq_{h r} Y, X \leq_{m l r} Y$ and $X \leq_{s t} Y$, which completes the proof of Proposition 8.

\subsection{Rényi Entropy}

To quantify the amount of information (such as the diversity, uncertainty, or randomness) contained in a random sample drawn from a population, the entropy is utilized. It may be noted that a large value of entropy indicates that the data contains greater uncertainty. Several studies have applied entropy in the fields of physics, probability and statistics, communication theory, economics, 
among others. Therefore, we derive one of the commonly used entropies, namely the Rényi entropy. For $\rho>0$ and $\rho \neq 1$, the Rényi entropy due to [19] is defined for a continuous random variable as

$$
E_{\rho}=\frac{1}{1-\rho} \log \left(\int_{0}^{\infty} f_{P H}^{\rho}(x) \mathrm{d} x\right)
$$

Using the pdf (3) in (53), we obtain

$$
\begin{aligned}
& E_{\rho}=\frac{1}{1-\rho} \log \left[\int_{0}^{\infty}\left(\frac{\beta \theta^{6}}{\alpha \theta^{5}+120}\left(\alpha+\frac{\theta}{6} x_{i}^{6 \beta}\right) x_{i}^{\beta-1} \mathrm{e}^{-\theta x_{i}^{\beta}}\right)^{\rho} \mathrm{d} x\right] \\
&=\frac{1}{1-\rho} \log \left[\left(\frac{\beta \theta^{6}}{\alpha \theta^{5}+120}\right)^{\rho} \int_{0}^{\infty}\left(\alpha+\frac{\theta}{6} x^{6 \beta}\right)^{\rho} x^{\rho(\beta-1)} \mathrm{e}^{-\theta \rho x^{\beta}} \mathrm{d} x\right] \\
&=\frac{1}{1-\rho} \log \left[\sum_{j=1}^{\rho}\left(\begin{array}{l}
\rho \\
j
\end{array}\right) \frac{\theta^{6 \rho+j} \alpha^{\rho-j} \beta^{\rho}}{6^{j}\left(\alpha \theta^{5}+120\right)^{\rho}} \int_{0}^{\infty} x^{6 \beta+\rho(\beta-1)} \mathrm{e}^{-\theta \rho x^{\beta}} \mathrm{d} x\right] \\
& \frac{1}{1-\rho} \log \left[\sum_{j=1}^{\rho}(\rho) \frac{\left.6^{-j} \theta^{6 \rho+j} \alpha^{\rho-j} \beta^{\rho-1} \int_{0}^{\infty} y^{(6 j+\rho-1 / \rho+1 / \beta)-1} \mathrm{e}^{-\theta \rho y} \mathrm{~d} y\right]}{\left(\alpha \theta^{5}+120\right)^{\rho}}\right] y=x^{\beta} \\
& E_{\rho}= \frac{1}{1-\rho} \log \left[\sum_{j=1}^{\rho}\left(\begin{array}{l}
\rho \\
j
\end{array}\right) \frac{6^{-j} \theta^{6 \rho+j} \alpha^{\rho-j} \beta^{\rho-1}}{\left(\alpha \theta^{5}+120\right)^{\rho}} \frac{\Gamma(6 j+\rho-1 / \rho+1 / \beta)}{(\theta \rho)^{6 j+\rho-1 / \rho+1 / \beta}}\right]
\end{aligned}
$$

\subsection{Distribution of Order Statistics for the PH Distribution}

Suppose $X_{(1)}<X_{(2)}<\cdots<X_{(n)}$ constitutes the order statistics for a random sample $X_{1}, X_{2}, \cdots, X_{n}$ drawn from the power Hamza distribution with pdf (3) and cdf (7). Then the pdf of the $r$ th order statistic $X_{(r)}$ can be written as

$$
\begin{aligned}
& f_{X_{(r)}}(x)=\frac{n !}{(r-1) !(n-r) !} f_{P H}(x)\left(F_{P H}(x)\right)^{r-1}\left(1-F_{P H}(x)\right)^{n-r} ; r=1,2, \cdots, n \\
& =\frac{\beta \theta^{6} n !}{\left(\alpha \theta^{5}+120\right)(r-1) !(n-r) !} \sum_{u=0}^{n-r}(-1)^{u}\left(\begin{array}{c}
n-r \\
u
\end{array}\right) x^{\beta-1} \mathrm{e}^{-\theta x^{\beta}}\left(\alpha+\frac{\theta}{6} x^{6 \beta}\right) \\
& \times\left[1-\left(1+\frac{\theta x^{\beta}\left(\theta^{5} x^{5 \beta}+6 \theta^{4} x^{4 \beta}+30 \theta^{3} x^{3 \beta}+120 \theta^{2} x^{2 \beta}+360 \theta x^{\beta}+720\right)}{\left(\alpha \theta^{5}+120\right)}\right) \mathrm{e}^{-\theta x^{\beta}}\right]^{r+u-1}
\end{aligned}
$$

Putting $r=1$ into (55) gives the pdf of the first order statistic $X_{(1)}$ as

$$
\begin{aligned}
& f_{X_{(1)}}(x)=\frac{n \beta \theta^{6}}{\alpha \theta^{5}+120} x^{\beta-1} \mathrm{e}^{-\theta x^{\beta}}\left(\alpha+\frac{\theta}{6} x^{6 \beta}\right) \\
& \times\left[\left(1+\frac{\theta x^{\beta}\left(\theta^{5} x^{5 \beta}+6 \theta^{4} x^{4 \beta}+30 \theta^{3} x^{3 \beta}+120 \theta^{2} x^{2 \beta}+360 \theta x^{\beta}+720\right)}{\left(\alpha \theta^{5}+120\right)}\right) \mathrm{e}^{-\theta x^{\beta}}\right]^{n-1}
\end{aligned}
$$

Putting $r=n$ into (55) gives the pdf of the $n$th order statistic $X_{(n)}$ as 


$$
\begin{aligned}
& f_{X_{(n)}}(x)=\frac{n \beta \theta^{6}}{\alpha \theta^{5}+120} x^{\beta-1} \mathrm{e}^{-\theta x^{\beta}}\left(\alpha+\frac{\theta}{6} x^{6 \beta}\right) \\
& \times\left(1-\left[1+\frac{\theta x^{\beta}\left(\theta^{5} x^{5 \beta}+6 \theta^{4} x^{4 \beta}+30 \theta^{3} x^{3 \beta}+120 \theta^{2} x^{2 \beta}+360 \theta x^{\beta}+720\right)}{\left(\alpha \theta^{5}+120\right)}\right] \mathrm{e}^{-\theta x^{\beta}}\right)^{n-1}
\end{aligned}
$$

\section{Maximum Likelihood Estimators of the Power Hamza Distribution}

Let $X_{1}, X_{2}, \cdots, X_{n}$ denote a random sample of size $n$ from the PH distribution having parameters $\theta, \alpha$ and $\beta$. To estimate the parameters $\theta, \alpha$ and $\beta$ using the maximum likelihood method, we define the likelihood function of the random sample form the $\mathrm{PH}$ distribution as

$$
L(\theta, \alpha, \beta)=\prod_{i=1}^{n} f\left(x_{i} ; \theta, \alpha, \beta\right)=\left(\frac{\beta \theta^{6}}{\alpha \theta^{5}+120}\right)^{n} \mathrm{e}^{-\theta \sum_{i=1}^{n} x_{i}^{\beta}} \prod_{i=1}^{n}\left(\alpha+\frac{\theta}{6} x_{i}^{6 \beta}\right) x_{i}^{\beta-1}
$$

Taking the natural $\log$ of (58), we obtain the log-likelihood function as

$$
\begin{aligned}
\ln L(\theta, \alpha, \beta)= & n\left[\ln (\beta)+6 \ln (\theta)-\ln \left(\alpha \theta^{5}+120\right)\right]+\sum_{i=1}^{n} \ln \left(\alpha+\frac{\theta}{6} x_{i}^{6 \beta}\right) \\
& +(\beta-1) \sum_{i=1}^{n} x_{i}-\theta \sum_{i=1}^{n} x_{i}^{\beta}
\end{aligned}
$$

Differentiating (59) with respect to $\theta, \alpha$ and $\beta$ respectively and equating the resulting derivatives to zero, one obtains

$$
\begin{gathered}
\frac{\partial \ln L(\theta, \alpha, \beta)}{\partial \theta}=\frac{6 n}{\theta}-\frac{5 n \alpha \theta^{4}}{\alpha \theta^{5}+120}+\sum_{i=1}^{n} \frac{x_{i}^{6 \beta}}{6 \alpha+\theta x_{i}^{6 \beta}}-\sum_{i=1}^{n} x_{i}^{\beta}=0 \\
\frac{\partial \ln L(\theta, \alpha, \beta)}{\partial \alpha}=\frac{-n \theta^{5}}{\alpha \theta^{5}+120}+\sum_{i=1}^{n} \frac{6}{6 \alpha+\theta x_{i}^{6 \beta}}=0 \\
\frac{\partial \ln L(\theta, \alpha, \beta)}{\partial \beta}=\frac{n}{\beta}+6 \theta \sum_{i=1}^{n} \frac{x_{i}^{6 \beta} \ln \left(x_{i}\right)}{6 \alpha+\theta x_{i}^{6 \beta}}+\sum_{i=1}^{n} \ln \left(x_{i}\right)-\theta \sum_{i=1}^{n} x_{i}^{\beta} \ln \left(x_{i}\right)=0
\end{gathered}
$$

The above non-linear systems of equations are solved by numerical iteration technique and maximum likelihood estimates are obtained. Since the maximum likelihood estimates for $\theta, \alpha$ and $\beta$ are not in closed form we use the large sample behaviour of maximum likelihood estimators to obtain the confidence intervals for model parameters.

\section{Asymptotic Confidence Intervals of the Power Hamza Distribution}

In this section, we present the asymptotic confidence intervals for the parameters of the $\mathrm{PH}$ distribution. Let $\hat{\boldsymbol{\psi}}=(\hat{\theta}, \hat{\alpha}, \hat{\beta})^{\mathrm{T}}$ be the maximum likelihood estimate of $\boldsymbol{\psi}=(\theta, \alpha, \beta)^{\mathrm{T}}$. Under the conditions that the parameters are in the interior of the parameter space, but not on the boundary, the asymptotic distribution of $\sqrt{n}(\hat{\boldsymbol{\psi}}-\boldsymbol{\psi})$ is $N_{3}\left(\mathbf{0}, \boldsymbol{I}^{-1}(\boldsymbol{\psi})\right)$, where $\boldsymbol{I}(\boldsymbol{\psi})$ is the expected Fisher information matrix. The asymptotic behaviour of the expected information ma- 
trix can be approximated by the observed information matrix, denoted by $\boldsymbol{I}_{n}(\hat{\boldsymbol{\psi}})$. The observed information matrix of the power Hamza distribution is given by

$$
\boldsymbol{I}_{n}(\hat{\boldsymbol{\psi}})=\left(\begin{array}{llll}
\frac{\partial^{2} \ln L(\theta, \alpha, \beta)}{\partial \theta^{2}} & \frac{\partial^{2} \ln L(\theta, \alpha, \beta)}{\partial \hat{\theta} \partial \hat{\alpha}} & \frac{\partial^{2} \ln L(\theta, \alpha, \beta)}{\partial \hat{\theta} \partial \hat{\beta}} \\
\frac{\partial^{2} \ln L(\theta, \alpha, \beta)}{\partial \hat{\alpha} \partial \hat{\theta}} & \frac{\partial^{2} \ln L(\theta, \alpha, \beta)}{\partial \hat{\alpha}^{2}} & \frac{\partial^{2} \ln L(\theta, \alpha, \beta)}{\partial \hat{\alpha} \partial \hat{\beta}} \\
\frac{\partial^{2} \ln L(\theta, \alpha, \beta)}{\partial \hat{\beta} \partial \hat{\theta}} & \frac{\partial^{2} \ln L(\theta, \alpha, \beta)}{\partial \hat{\beta} \partial \hat{\alpha}} & \frac{\partial^{2} \ln L(\theta, \alpha, \beta)}{\partial \hat{\beta}^{2}}
\end{array}\right)
$$

Thus,

$$
\boldsymbol{I}_{n}^{-1}(\hat{\boldsymbol{\psi}})=(n \boldsymbol{I}(\hat{\boldsymbol{\psi}}))^{-1}=\left(\begin{array}{ccc}
\operatorname{var}(\hat{\theta}) & \operatorname{cov}(\hat{\theta}, \hat{\alpha}) & \operatorname{cov}(\hat{\theta}, \hat{\beta}) \\
\operatorname{cov}(\hat{\alpha}, \hat{\theta}) & \operatorname{var}(\hat{\alpha}) & \operatorname{cov}(\hat{\alpha}, \hat{\beta}) \\
\operatorname{cov}(\hat{\beta}, \hat{\theta}) & \operatorname{cov}(\hat{\beta}, \hat{\alpha}) & \operatorname{var}(\hat{\beta})
\end{array}\right)
$$

Taking the second order derivatives of (59) with respect to $\theta, \alpha$ and $\beta$ are, respectively, we obtain the entries of (63) as follows

$$
\begin{aligned}
& \frac{\partial^{2} \ln L(\theta, \alpha, \beta)}{\partial \theta^{2}}=-\frac{6 n}{\theta}+\frac{5 n \alpha \theta^{3}\left(\alpha \theta^{5}-480\right)}{\left(\alpha \theta^{5}+120\right)^{2}}-\sum_{i=1}^{n} \frac{x_{i}^{12 \beta}}{\left(6 \alpha+\theta x_{i}^{6 \beta}\right)^{2}} \\
& \frac{\partial^{2} \ln L(\theta, \alpha, \beta)}{\partial \alpha^{2}}=\frac{n \theta^{10}}{\left(\alpha \theta^{5}+120\right)^{2}}-\sum_{i=1}^{n} \frac{36}{\left(6 \alpha+\theta x_{i}^{6 \beta}\right)^{2}} \\
& \frac{\partial^{2} \ln L(\theta, \alpha, \beta)}{\partial \beta^{2}}=-\frac{n}{\beta^{2}}+216 \alpha \theta \sum_{i=1}^{n} \frac{x_{i}^{6 \beta} \ln ^{2}\left(x_{i}\right)}{\left(6 \alpha+\theta x_{i}^{6 \beta}\right)^{2}}-\theta \sum_{i=1}^{n} x_{i}^{\beta} \ln ^{2}\left(x_{i}\right) \\
& \frac{\partial^{2} \ln L(\theta, \alpha, \beta)}{\partial \alpha \partial \theta}=\frac{-600 n \theta^{4}}{\left(\alpha \theta^{5}+120\right)^{2}}-6 \sum_{i=1}^{n} \frac{x_{i}^{6 \beta}}{\left(6 \alpha+\theta x_{i}^{6 \beta}\right)^{2}} \\
& \frac{\partial^{2} \ln L(\theta, \alpha, \beta)}{\partial \beta \partial \theta}=36 \alpha \sum_{i=1}^{n} \frac{x_{i}^{6 \beta} \ln \left(x_{i}\right)}{\left(6 \alpha+\theta x_{i}^{6 \beta}\right)^{2}}-\sum_{i=1}^{n} x_{i}^{\beta} \ln \left(x_{i}\right) \\
& \frac{\partial^{2} \ln L(\theta, \alpha, \beta)}{\partial \alpha \partial \beta}=-36 \sum_{i=1}^{n} \frac{x_{i}^{6 \beta} \ln \left(x_{i}\right)}{\left(6 \alpha+\theta x_{i}^{6 \beta}\right)^{2}}
\end{aligned}
$$

The expectations in the Fisher information matrix can be obtained numerically. The multivariate normal distribution with mean vector $(0,0,0)^{\mathrm{T}}$ and covariance matrix $I^{-1}(\psi)$ can be used to construct confidence intervals for the model parameters. The approximate $100(1-\eta) \%$ two-sided confidence intervals for $\theta, \alpha$ and $\beta$ are determined by

$$
\hat{\theta} \pm Z_{\eta / 2} \sqrt{\operatorname{var}(\hat{\theta})}, \hat{\alpha} \pm Z_{\eta / 2} \sqrt{\operatorname{var}(\hat{\alpha})} \text { and } \hat{\beta} \pm Z_{\eta / 2} \sqrt{\operatorname{var}(\hat{\beta})}
$$

respectively, where $Z_{\eta / 2}$ is the upper $(\eta / 2)$ th percentile of a standard normal distribution. 


\section{Applications}

In this section, we provide an application to real data set to demonstrate the importance and flexibility of the PH distribution.

The data set is on the breaking strength of carbon fibres of $50 \mathrm{~mm}$ length (GPa). The data has been previously used by [20] and [21]. The data is as follows:

$0.39,0.85,1.08,1.25,1.47,1.57,1.61,1.61,1.69,1.80,1.84,1.87,1.89,2.03,2.03$, $2.05,2.12,2.35,2.41,2.43,2.48,2.50,2.53,2.55,2.55,2.56,2.59,2.67,2.73,2.74$, $2.79,2.81,2.82,2.85,2.87,2.88,2.93,2.95,2.96,2.97,3.09,3.11,3.11,3.15,3.15$, $3.19,3.22,3.22,3.27,3.28,3.31,3.31,3.33,3.39,3.39,3.56,3.60,3.65,3.68,3.70$, $3.75,4.20,4.38,4.42,4.70,4.90$.

We fitted the $\mathrm{PH}$ distribution to the data set by using the method of maximum likelihood and the results are compared with five other competitive lifetime distributions namely,

1) Hamza distribution (HD) defined in Equation (2),

2) Weighted Weibull distribution (WWD):

$$
f(x ; \theta, \beta)=\frac{\theta \beta^{c+1} x^{\beta(c+1)-1} \mathrm{e}^{-\theta x^{\beta}}}{\Gamma(c+1)} ; x>0, \theta>0, \beta>0, c>-1,
$$

3) Two-Parameter Weibull distribution (TPWD):

$$
f(x ; \theta, \beta)=\theta \beta x^{\beta-1} \mathrm{e}^{-\theta x^{\beta}} ; x>0, \theta>0, \beta>0,
$$

4) Pareto distribution (PD):

$$
f(x ; \alpha)=\frac{\alpha x_{m}^{\alpha}}{x_{m}^{\alpha+1}} ; x>0, \alpha>0,
$$

5) Exponential distribution (ED):

$$
f(x ; \theta)=\theta \mathrm{e}^{-\theta x} ; x>0, \theta>0 .
$$

We used the goodness-of-fit test based on the Kolmogorov-Smirnov test due to ([22] [23] [24] [25]) with its corresponding p-value to verify that the data set under consideration actually follow the proposed distribution. The computational formula for this goodness-of-fit test is given by

$$
\mathrm{KS}=\max \left\{\frac{i}{n}-\hat{F}\left(x_{(i)}\right), \hat{F}\left(x_{(i)}\right)-\frac{i-1}{n}\right\}
$$

where $\hat{F}\left(x_{i}\right)$ is the estimated distribution function under the ordered data. Since there is more than one distribution to be compared, the distribution with the largest KS p-value will be more appropriate to fit the given sample.

We shall also determine the appropriate model from among all models compared for the real data set by considering three discrimination criteria, based on the log-likelihood function evaluated at the maximum likelihood estimates, the Akaike information criterion (AIC) due to [26] and the Bayesian information criterion (BIC) due to [27], respectively. To compute the AIC and BIC, the following formulae are used 
Table 1. Parameter estimates, standard errors, log-likelihood values and goodness-of-fit measures.

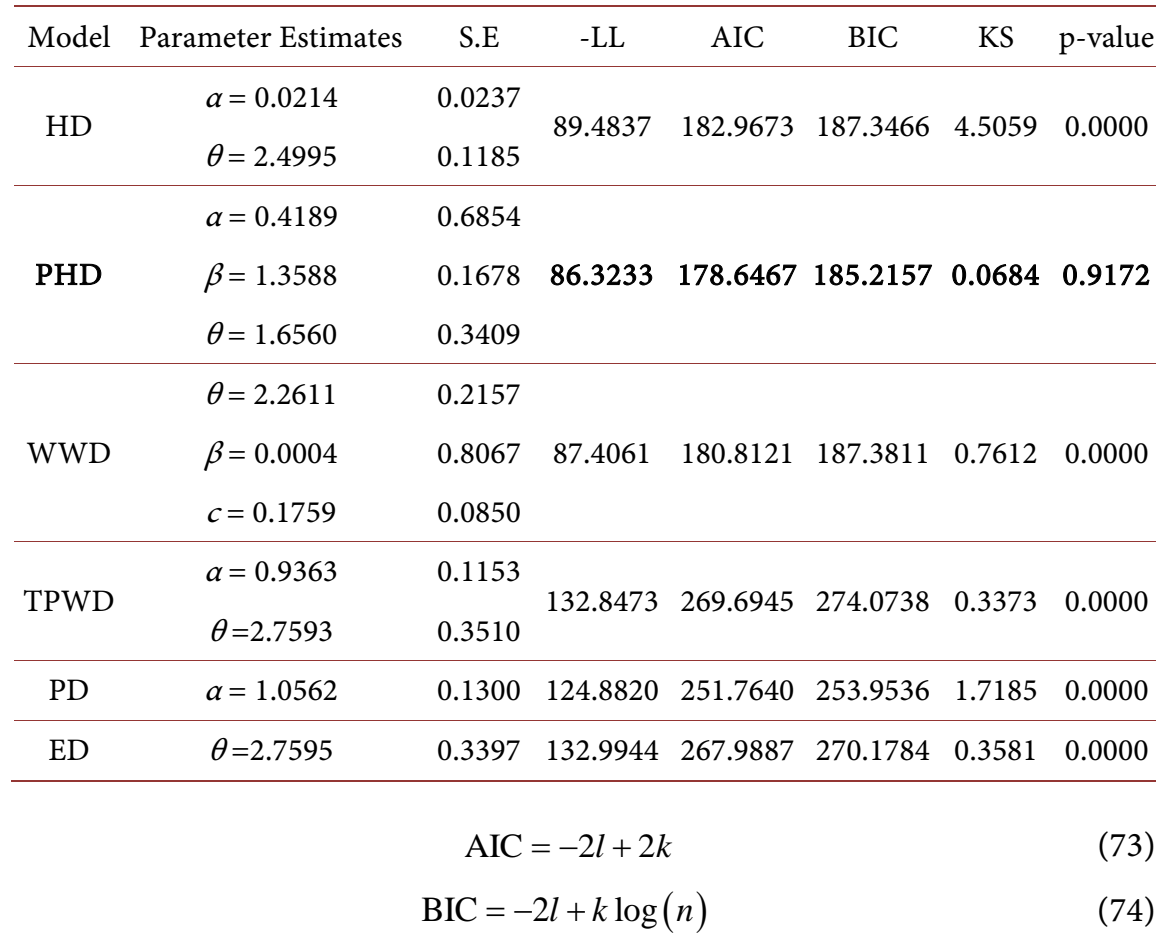

where $I$ denotes the log-likelihood function evaluated at the maximum likelihood estimates, $k$ is the number of parameters in the statistical model and $n$ is the sample size of the fitted data respectively. All the computations for (72)-(74) were performed using R software. Generally, for the given data-sets, we consider a distribution to be best among all competing distributions if it has smallest AIC value, the smallest BIC value, the smallest log-likelihood value and the largest p-value.

As shown in Table 1, the $\mathrm{PH}$ distribution has the largest KS p-value and smallest AIC, BIC and log-likelihood values as compared to other fitted distributions. Hence, the $\mathrm{PH}$ distribution is better than the other distributions in Table 1 for fitting the data under consideration.

\section{Conclusion}

This study introduced a new distribution, called the power Hamza distribution using power transformation method. The contribution of this paper has to do with addition of skewness to the Hamza distribution, which depends only on one parameter. The density function of the power Hamza distribution can take various forms depending on its shape parameter. The hazard rate function of the power Hamza distribution exhibits heavy-tailed shape, upside-down bathtub shape and J-shape, which implies that the distribution can be used for analyzing lifetime and survival time datasets. A detailed discussion of the properties of the proposed distribution has been given. Estimates of the three unknown parameters of the PH distribution are obtained using the maximum likelihood estima- 
tion method. The PH distribution was fitted to a real dataset and compared to five distributions and the results showed that the proposed distribution outperformed all of them in modelling the data under consideration.

\section{Acknowledgements}

The authors are thankful to the editor and reviewers of this article for providing very useful comments which led to the improvement of this paper.

\section{Authors' Contribution}

All authors contributed equally in developing the article.

\section{Funding Statement}

This research did not receive any specific grant from funding agencies in the public, commercial, or not-for-profit sectors.

\section{Conflicts of Interest}

The authors declare no conflicts of interest regarding the publication of this paper.

\section{References}

[1] Aijaz, A., Jallal, M., Ain Ul, S.Q. and Tripathi, R. (2020) The Hamza Distribution with Statistical Properties and Applications. Asian Journal of Probability and Statistics, 8, 28-42. https://doi.org/10.9734/ajpas/2020/v8i130198

[2] Lindley, D.V. (1958) Fiducial Distributions and Bayes. Journal of the Royal Statistical Society, Series B, 20, 102-107. https://doi.org/10.1111/j.2517-6161.1958.tb00278.x

[3] Shanker, R. and Shukla, K.K. (2017) Ishita Distribution and Its Applications. Biometrics \& Biostatistics International Journal, 5, 1-9. https://doi.org/10.15406/bbij.2017.05.00126

[4] Shukla, K.K. (2018) Pranav Distribution with Properties and Its Applications. Biometrics and Biostatistics International Journal, 7, 244-254. https://doi.org/10.15406/bbij.2018.07.00215

[5] Ghitany, M.E., Al-Mutairi, D.K., Balakrishnan, N. and Al-Enezi, L.J. (2013) Power Lindley Distribution and Associated Inference. Computational Statistics and Data Analysis, 64, 20-33. https://doi.org/10.1016/j.csda.2013.02.026

[6] Shanker, R. and Shukla, K.K. (2017) Power Akash Distribution and Its Applications. Journal of Applied Quantitative Methods, 12, 1-1012.

[7] Shanker, R. and Shukla, K.K. (2017) Power Shanker Distribution and Its Application. Turkiye Klinikleri Journal of Biostatistics, 9, 175-187. https://doi.org/10.5336/biostatic.2017-56601

[8] Shukla, K.K. and Shanker, R. (2018) Power Ishita Distribution and Its Applications to Model Lifetime Data. Statistics in Transition New Series, 19, 135-148. https://doi.org/10.21307/stattrans-2018-008

[9] Shanker, R. and Shukla, K.K. (2018) A Two-Parameter Power Aradhana Distribution with Properties and Application. Indian Journal of Industrial and Applied 
Mathematics, 9, 210-220. https://doi.org/10.5958/1945-919X.2018.00016.6

[10] Abebe, B., Tesfay, M., Eyob, T. and Shanker, R. (2019) A Two-Parameter Power Rama Distribution with Properties and Applications. Biometrics and Biostatistics International Journal, 8, 6-11. https://doi.org/10.15406/bbij.2019.08.00262

[11] Abebe, B., Tesfay, M., Eyob, T. and Shanker, R. (2019) A Two-Parameter Power Garima Distribution with Properties and Applications. Annals of Biostatistics and Biometric Applications, 1, 1-7. https://doi.org/10.33552/ABBA.2019.01.000515

[12] Shukla, K.K. (2019) Power Pranav Distribution and Its Applications. Journal of Applied Quantitative Methods, 14, 1-13.

[13] Shanker, R. and Shukla, K.K. (2019) A Two-Parameter Power Sujatha Distribution with Properties and Application. International Journal of Mathematics and Statistics, 20, 11-22.

[14] Shukla, K.K. and Shanker, R. (2020) Power Prakaamy Distribution and Its Applications. International Journal of Computations and Theoretical Statistics, 7, 26-36.

[15] Hogg, R.V., McKean, J.W. and Craig, A.T. (2019) Introduction to Mathematical Statistics. 8th Edition, Pearson Education, Inc., London.

[16] Bonferroni, C.E. (1930) Elementi di Statistica Generale, Seeber, Firenze.

[17] Lorenz, M.O. (1905) Methods of Measuring the Concentration of Wealth. Publications of the American Statistical Association, 9, 209-219. https://doi.org/10.2307/2276207

[18] Shaked, M. and Shanthikumar, J.G. (1994) Stochastic Orders and Their Applications. Academic Press, Boston.

[19] Rényi, A. (1961) On Measure of Entropy and Information. In: Proceedings of the 4th Berkeley Symposium on Mathematical Statistics and Probability, Vol. 1, University of California Press, Berkeley, 547-561.

[20] Cordeiro, G.M. and Lemonte, A.J. (2011) The $\beta$-Birnbaum-Saunders Distribution: An Improved Distribution for Fatigue Life Modelling. Computational Statistics and Data Analysis, 55, 1445-1461. https://doi.org/10.1016/j.csda.2010.10.007

[21] Al-Aqtash, R., Lee, C. and Famoye, F. (2014) Gumbel-Weibull Distribution: Properties and Applications. Journal of Modern Applied Statistical Methods, 13, 201-225. https://doi.org/10.22237/jmasm/1414815000

[22] Kolmogorov, A. (1933) Sulla determinazione empirica di una lgge di distribuzione. Giornale dell Istituto Italiano degli Attuari, 4, 83-91.

[23] Smirnoff, N. (1939) Sur les écarts de la courbe de distribution empirique. Matematicheskii Sbornik, 48, 3-26.

[24] Scheffé, H. (1943) Statistical Inference in the Non-Parametric Case. The Annals of Mathematical Statistics, 14, 305-332. https://doi.org/10.1214/aoms/1177731355

[25] Wolfowitz, J. (1949) Non-Parametric Statistical Inference. In: Proceedings of the Berkeley Symposium on Mathematical Statistics and Probability, University of California Press, Berkeley, 93-113.

[26] Akaike, H. (1974) A New Look at the Statistical Model Identification. IEEE Transactions on Automatic Control, 19, 716-723.

https://doi.org/10.1109/TAC.1974.1100705

[27] Schwarz, G. (1978) Estimating the Dimension of a Model. Annals of Statistics, 6, 461-464. https://doi.org/10.1214/aos/1176344136 